\title{
Serum IgG subclass levels and risk of exacerbations and hospitalizations in patients with COPD
}

Fernando Sergio Leitao Filho ${ }^{1,2,3}$, Seung Won Ra ${ }^{1,4}$, Andre Mattman ${ }^{5}$, Robert S. Schellenberg ${ }^{1,2,3}$, Gerard J. Criner ${ }^{6}$, Prescott G. Woodruff', Stephen C. Lazarus ${ }^{7}$, Richard Albert ${ }^{8}$, John E. Connett ${ }^{9}$, Meilan K. Han $^{10}$, Fernando J. Martinez ${ }^{11}$, Janice M. Leung ${ }^{1,2}$, S. F. Paul Man ${ }^{1,2}$, Shawn D. Aaron ${ }^{12 \dagger}$, Robert M. Reed ${ }^{13 \dagger}$, Don D. $\operatorname{Sin}^{1,2^{*}+}$ and for the Canadian Respiratory Research Network (CRRN)

\begin{abstract}
Background: The literature is scarce regarding the prevalence and clinical impact of IgG subclass deficiency in COPD. We investigated the prevalence of $\operatorname{lgG}$ subclass deficiencies and their association with exacerbations and hospitalizations using subjects from two COPD cohorts.

Methods: We measured IgG subclass levels using immunonephelometry in serum samples from participants enrolled in two previous COPD trials: Macrolide Azithromycin for Prevention of Exacerbations of COPD (MACRO; $n=976)$ and Simvastatin for the Prevention of Exacerbations in Moderate-to-Severe COPD (STATCOPE; $n=653$ ). All samples were collected from clinically stable participants upon entry into both studies. IgG subclass deficiency was diagnosed when IgG subclass levels were below their respective lower limit of normal: $\operatorname{lgG} 1<2.8 \mathrm{~g} / \mathrm{L} ; \operatorname{lgG} 2<1.15 \mathrm{~g} / \mathrm{L} ; \operatorname{lgG} 3<0.24 \mathrm{~g} / \mathrm{L}$; and $\operatorname{lgG} 4<0.052 \mathrm{~g} / \mathrm{L}$. To investigate the impact of $\mathrm{lgG}$ subclass levels on time to first exacerbation or hospitalization, we log-transformed IgG levels and performed Cox regression models, with adjustments for confounders.
\end{abstract}

Results: One or more lgG subclass deficiencies were found in 173 (17.7\%) and 133 (20.4\%) participants in MACRO and STATCOPE, respectively. Lower lgG1 or lgG2 levels resulted in increased risk of exacerbations with adjusted hazard ratios (HR) of $1.30(95 \% \mathrm{Cl}, 1.10-1.54, p<0.01)$ and $1.19(95 \% \mathrm{Cl}, 1.05-1.35, p<0.01)$, respectively in the MACRO study, with STATCOPE yielding similar results. Reduced lgG1 or lgG2 levels were also associated with increased risk of hospitalizations: the adjusted HR for lgG1 and lgG2 was 1.52 (95\% Cl: 1.15-2.02, $p<0.01)$ and $1.33(95 \% \mathrm{Cl}, 1.08-1.64, p<0.01)$, respectively for the MACRO study; in STATCOPE, only lgG2 was an independent predictor of hospitalization. In our multivariate Cox models, lgG3 and lgG4 levels did not result in significant associations for both outcomes in either MACRO or STATCOPE cohorts.

Conclusions: Approximately 1 in 5 COPD patients had one or more lgG subclass deficiencies. Reduced lgG subclass levels were independent risk factors for both COPD exacerbations (lgG1 and lgG2) and hospitalizations (lgG2) in two COPD cohorts.

Trial registration: This study used serum samples from participants of the MACRO (NCT00325897) and STATCOPE (NCT01061671) trials.

Keywords: lgG, lgG subclass deficiency, COPD, Exacerbation, Hospitalization

\footnotetext{
* Correspondence: don.sin@hli.ubc.ca

${ }^{\dagger}$ Equal contributors

'Centre for Heart Lung Innovation, St. Paul's Hospital, Vancouver, BC V6Z 1Y6,

Canada

${ }^{2}$ Department of Medicine (Division of Respiratory Medicine), University of

British Columbia, Vancouver, BC, Canada

Full list of author information is available at the end of the article
} 


\section{Background}

Chronic obstructive pulmonary disease (COPD) is one of the leading causes of morbidity and mortality worldwide, projected to become the third leading cause of death in 2020 [1]. The course of COPD is punctuated by episodes of acute exacerbations, which result in worse quality of life, accelerated disease progression, and increased risk of mortality $[1,2]$. Hospitalizations due to severe exacerbations are associated with poor survival, with a 5-year mortality rate of $55 \%$ based on a previous study [3]. Respiratory tract infections are major triggers of COPD exacerbations $[1,4]$.

There is increasing evidence that immunoglobulins (Ig) play a crucial role in preventing infections, particularly sinopulmonary infections which likely relate to the continuous exposure of the anatomy involved to a vast range of pathogens [5-9]. Immunoglobulin G (IgG) is the predominant Ig class, corresponding to $75-80 \%$ of total Ig levels in blood, and consisting of four subclasses (IgG1, IgG2, IgG3, and IgG4) [10, 11]. The primary mechanisms by which IgG inactivates or eliminates pathogens involve activation of the complement system and opsonization of pathogens [12]. These processes result in target cell lysis and enhanced phagocytosis, respectively $[10,13]$.

Recently, we demonstrated that hypogammaglobulinemia (defined as total IgG levels $<7.0 \mathrm{~g} / \mathrm{L}$ ) was present in approximately 1 out of 4 patients with moderate to severe COPD [14]. More importantly, those patients with reduced IgG levels demonstrated $50 \%$ to $100 \%$ higher risk for both exacerbations and hospitalizations. The relationship between various subclasses of IgG and risk of exacerbations, however, remains unknown, as only a few articles with a number of limitations have described the frequency of IgG subclass deficiencies in COPD[15-17] and no prior studies have evaluated their impact on hospitalizations. Considering that COPD is a heterogeneous disease with several clinical phenotypes [18], we analyzed samples from patients who were at increased risk for exacerbations, and a second independent COPD cohort was also used to replicate our findings.

\section{Methods}

\section{Study design}

This is a retrospective study which used samples from participants of two COPD cohorts: Macrolide Azithromycin for Prevention of Exacerbations of COPD (MACRO - First cohort; ClinicalTrials.gov number: NCT00325897) [19] and Simvastatin for the Prevention of Exacerbations in Moderate-to-Severe COPD (STATCOPE - Replication cohort; ClinicalTrials.gov number: NCT01061671) [20]. Briefly, the MACRO trial tested azithromycin (250 mg daily), a macrolide antibiotic with immunomodulatory and antibacterial effects, whereas the STATCOPE trial investigated simvastatin (40 mg daily), compared to placebo for the prevention of COPD exacerbations. Both trials were double- blinded and placebo-controlled intervention studies. All participants had a diagnosis of COPD, aged 40 years or older, were current or former smokers ( $\geq 10$ pack-year smoking history), and had a forced expiratory volume in $1 \mathrm{~s}\left(\mathrm{FEV}_{1}\right)<$ $80 \%$ of predicted values after bronchodilator use. Additionally, all subjects had to meet at least one of the following inclusion criteria: use of supplemental oxygen, previous treatment with systemic glucocorticoids or antibiotic agents for respiratory problems, or a history of an emergency department visit or hospitalization for COPD exacerbation.

\section{Exacerbation and hospitalization data}

In both cohorts, the number and severity of exacerbations that occurred during follow-up were captured. The following scale was used to grade the severity of exacerbations: 1) mild - treatment conducted at home, with or without contacting a health care provider; 2) moderate - treatment requiring a visit to the emergency department; 3) severe treatment requiring hospitalization; and 4) very severe - in which patients needed intensive care unit (ICU) admission. COPD-related hospitalizations were identified by capturing all events graded as severe and very severe exacerbations.

\section{IgG measurements}

Baseline blood samples were collected from clinically stable participants upon entry into both studies. A total of 978 and 654 samples from the MACRO and STATCOPE cohorts, respectively, were available. Two samples from the MACRO trial (azithromycin arm) and one sample from the STATCOPE trial (placebo arm) were of insufficient quantity to allow measurements of all IgG subclasses and were thus not included in the analysis. In total, IgG levels were measured in 1629 samples, 976 from participants of MACRO (468 in the azithromycin arm; 508 in the placebo arm) and 653 from participants of STATCOPE (322 in the simvastatin arm; 331 in the placebo arm). Serum IgG subclass levels were measured using Binding Site IgG subclass (IgG1-4) reagents (Birmingham, UK) by immunonephelometry on a Siemens BNII nephelometer (BNII; Erlangen, Germany). All samples were processed in the clinical laboratory at St. Paul's Hospital, Vancouver, British Columbia, Canada. The following normal ranges for IgG subclasses in adults were used: IgG1, 2.80-8.00 g/L; IgG2, 1.15-5.70 g/L; IgG3, 0.24-1.25 g/L; IgG4, 0.052-1.25 g/L [21]. These ranges were obtained from a healthy population and include mean levels \pm 2 standard deviations [22]. IgG subclass deficiency was diagnosed when IgG levels were below their respective lower limit of normal for each subclass: IgG1 $<2.80 \mathrm{~g} / \mathrm{L}, \operatorname{IgG} 2<$ $1.15 \mathrm{~g} / \mathrm{L}, \operatorname{IgG} 3<0.24 \mathrm{~g} / \mathrm{L}$, and IgG4 < $0.052 \mathrm{~g} / \mathrm{L}$.

\section{Statistical analysis}

IgG subclass levels were not normally distributed, and thus are presented as median and interquartile range. Categorical 
variables are shown as number and percentages. We used the Mann-Whitney U test and the Pearson's Chi-square test to compare the IgG subclass levels and the frequency of IgG subclass deficiencies between the two cohorts, respectively. The Mann-Whitney $U$ test was also applied to analyze the IgG subclass levels according to the exacerbation status (participants with zero or one exacerbation vs. participants with two or more exacerbations) and the occurrence of hospitalizations during follow-up. Additionally, we compared the rates of COPD exacerbations in person-years (expressed as the number of acute exacerbations divided by the duration of follow-up in years) between participants with IgG subclass levels below and above the lower limit of normal (LLN) using the Mann-Whitney U test.

To investigate the effect of IgG levels on time to first COPD exacerbation or hospitalization, we applied Cox proportional-hazards regression models to obtain a hazard ratio (HR) and its 95\% confidence intervals (CI) associated with these outcomes for each IgG subclass. In our Cox models, we adjusted for covariates that were considered biologically relevant to the outcomes of interest (exacerbations and hospitalizations) or that presented with a $p<0.2$ at the univariate analysis. The following variables were included in our multivariate models: IgG subclass levels (logtransformed), study drug (azithromycin in the derivation cohort, or simvastatin in the validation cohort), age, gender, ethnicity, smoking status, $\mathrm{FEV}_{1}$ (\% of predicted value), long-term oxygen therapy, inhaled corticosteroid therapy at baseline, and history of systemic steroids in the year prior to enrollment. To investigate any possible effect of steroids (as described above) on IgG subclass levels, we also included an interaction term in our multivariate models. For IgG subclasses that demonstrated significant associations in our time to event analyses, we also obtained the adjusted predicted probabilities of COPD exacerbations or hospitalizations (using logistic regression models) and calculated the Pearson's correlation coefficient between these probabilities and IgG-levels (log-transformed). SPSS version 22.0 for Windows (IBM Corp., Armonk, NY, USA) was used for data analysis. The level of statistical significance was set at $p<0.05$ for all tests.

\section{Results}

Characteristics of participants and prevalence of IgG deficiency The details of participant characteristics at baseline are presented in Table 1 . In MACRO, the participants were older $(65.5 \pm 8.6$ vs. $62.8 \pm 8.4$ years, $p<0.001)$, were more likely to be Caucasians $(82.6 \%$ vs. $77.9 \%, p=0.02)$ and had slightly lower $\mathrm{FEV}_{1}(39.8 \pm 15.7 \%$ vs. $41.5 \pm$ $17.8 \%, p=0.049$ ), and a higher rate of long-term domiciliary oxygen use $(60.1 \%$ vs. $48.7 \%, p<0.001)$ compared with participants enrolled in STATCOPE. Conversely, the prevalence of current smokers was higher in STATCOPE $(28.8 \%$ vs. $21.2 \%, p<0.001)$.
Total IgG and IgG subclass levels were similar between both cohorts (Table 2). Overall, 306 (18.8\%) participants in the merged cohort $(n=1629)$ presented with at least one IgG subclass deficiency (173 (17.7\%) and 133 (20.4\%) in MACRO and STATCOPE, respectively)(Table 2). IgG3 deficiency was the most frequent, whereas IgG1 deficiency was the least common. We also identified cases showing two or more IgG subclasses deficiencies simultaneously. The majority of participants identified with an IgG subclass deficiency were also diagnosed with hypogammaglobulinemia (defined as total $\operatorname{IgG}<7.0 \mathrm{~g} / \mathrm{L}$ ), especially participants presenting with IgG1 $(72 / 74, n=97.3 \%)$ or IgG2 $(69 / 93,74.2 \%)$ deficiencies. No differences regarding the frequency of IgG subclass deficiencies between both MACRO and STATCOPE cohorts were observed (Additional file 1: Table S1). The median and interquartile range for each IgG subclass according to the presence or absence of the related IgG subclass deficiency are shown in Additional file 2: Tables S2 (First and Replication cohorts) and Additional file 3: Table S3 (merged cohort).

\section{IgG subclass levels according to exacerbation status and hospitalizations}

In both cohorts, we detected significantly lower IgG1 and IgG2 levels among participants who developed two or more exacerbations during follow-up versus those who did not have any exacerbations or developed only one exacerbation (Additional file 4: Figure S1). Similar results were also observed in both cohorts according to hospitalization status, with participants who required hospitalizations during follow-up presenting with lower IgG1 and IgG2 subclass levels (Additional file 5: Figure S2).

Given the low frequency of IgG subclass deficiency $(<$ $10 \%)$ for all IgG subclasses in both cohorts, we merged both cohorts $(n=1629)$ and compared the rates of COPD exacerbations per person-year between participants according to the presence or not of IgG1-4 deficiencies. We observed significantly higher rates of exacerbations per person-year among participants with IgG1 deficiency (2.9 \pm 5.46 vs. $1.48 \pm 1.86, p<0.001)$ and IgG2 deficiency $(2.10 \pm$ 1.99 vs. $1.51 \pm 2.18, p=0.001)$ compared to their counterparts with normal IgG1 and IgG2 levels (Fig. 1). Additionally, the frequency of IgG1 and IgG2 deficiency was also significantly higher among recurrent exacerbators compared to participants who remained stable or presented with only one exacerbation during the follow-up: IgG1-43/ $644(6.7 \%)$ vs. $31 / 985$ (3.1\%), $p=0.001$; IgG2-47/644 (7.3\%) vs. $46 / 985(4.7 \%), p=0.025$.

\section{Impact of IgG subclass levels on time to first COPD exacerbation and hospitalization}

The adjusted HRs for having a COPD exacerbation increased significantly with progressively lower IgG1 and IgG2 levels in MACRO (HR: 1.30, 95\% CI: 1.10-1.54, $p=$ 0.002 ; HR: $1.19,95 \%$ CI: $1.05-1.35, p=0.006$, respectively) 
Table 1 Baseline characteristics of participants in the MACRO and STATCOPE cohorts

\begin{tabular}{llll}
\hline Variable & MACRO $(n=976)$ & STATCOPE $(n=653)$ & $P^{*}$ \\
\hline Age, years & $65.5 \pm 8.6$ & $62.8 \pm 8.4$ & $381(58.3): 272(41.7)$ \\
Gender (Male: Female) - no. (\%) & $584(59.8): 392(40.2)$ & $509(77.9)$ & 0.001 \\
Ethnicity (Caucasian) - no. (\%) & $806(82.6)$ & $27.1 \pm 6.8$ & 0.02 \\
BMl, kg/m & $27.7 \pm 6.2$ & $188(28.8)$ & 0.062 \\
Current Smokers - no. (\%) & $207(21.2)$ & $71.1 \pm 19.1$ & $<0.001$ \\
FVC, \% predicted & $70.2 \pm 18.1$ & $41.5 \pm 17.8$ & 0.29 \\
FEV 1 , \% predicted & $39.8 \pm 15.7$ & $318(48.7)$ & 0.049 \\
Long-term oxygen therapy - no. (\%) & $587(60.1)$ & $550(84.2)$ & $<0.001$ \\
Systemic steroid use in previous year - no. (\%) & $822(84.2)$ & $484(74.1)$ & 0.99 \\
Inhaled steroid use - no. (\%) & $764(78.3)$ & $5.26(2.70)$ & 0.06 \\
IgG1 (g/L) & $5.09(2.45)$ & $2.61(1.64)$ & 0.18 \\
IgG2 (g/L) & $2.57(1.72)$ & $0.63(0.51)$ & 0.82 \\
IgG3 (g/L) & $0.62(0.49)$ & $0.22(0.32)$ & 0.96 \\
IgG4 (g/L) & $0.21(0.28)$ & $8.84(4.00)$ & 0.99 \\
Total lgG (g/L) & $8.68(3.72)$ & 0.70 \\
\hline
\end{tabular}

Values expressed as means \pm SD. IgG levels expressed as median (interquartile range). *Student's t-test, Mann-Whitney U-test (for comparisons of IgG levels), or Chi-square test, as appropriate. Definition of abbreviations: $B M I$ body mass index, $F V C$ forced vital capacity, $F E V$ forced expiratory volume in one second, $I g G$ immunoglobulin $\mathrm{G}$

Table 2 Frequency of IgG subclass deficiency according to the presence or absence of associated hypogammaglobulinemia in the merged cohort (MACRO and STATCOPE cohorts combined)

\begin{tabular}{|c|c|}
\hline IgG abnormality & Merged Cohort $(n=1629)$ \\
\hline IgG1 deficiency - no. (\%) & $74(4.5 \%)$ \\
\hline With hypogammaglobulinemia - no. (\%) & $72(4.4 \%)$ \\
\hline No hypogammaglobulinemia - no. (\%) & $2(0.1 \%)$ \\
\hline lgG2 deficiency - no. (\%) & $93(5.7 \%)$ \\
\hline With hypogammaglobulinemia - no. (\%) & $69(4.2 \%)$ \\
\hline No hypogammaglobulinemia - no. (\%) & $24(1.5 \%)$ \\
\hline lgG3 deficiency - no. (\%) & $124(7.6 \%)$ \\
\hline With hypogammaglobulinemia - no. (\%) & $72(4.4 \%)$ \\
\hline No hypogammaglobulinemia - no. (\%) & $52(3.2 \%)$ \\
\hline IgG4 deficiency - no. (\%) & $114(7.0 \%)$ \\
\hline With hypogammaglobulinemia - no. (\%) & $73(4.5 \%)$ \\
\hline No hypogammaglobulinemia - no. (\%) & $41(2.5 \%)$ \\
\hline One or more IgG subclass deficiency - no. (\%) & $306(18.8 \%)$ \\
\hline With hypogammaglobulinemia - no. (\%) & $197(12.1 \%)$ \\
\hline No hypogammaglobulinemia - no. (\%) & $109(6.7 \%)$ \\
\hline Two IgG subclass deficiencies combined - no. (\%) & $47(2.9 \%)$ \\
\hline With hypogammaglobulinemia - no. (\%) & $37(2.3 \%)$ \\
\hline No hypogammaglobulinemia - no. (\%) & $10(0.6 \%)$ \\
\hline Three IgG subclass deficiencies combined - no. (\%) ${ }^{\mathrm{a}}$ & $20(1.2 \%)$ \\
\hline All IgG subclass deficiencies combined - no. (\%) ${ }^{a}$ & $4(0.2 \%)$ \\
\hline
\end{tabular}

The normal range for IgG levels in adults used in this analysis were: $\operatorname{lgG} 1,2.8-8.0 \mathrm{~g} / \mathrm{L} ; \mathrm{lgG}, 1.15-5.70 \mathrm{~g} / \mathrm{L} ; \mathrm{lgG}, 0.24-1.25 \mathrm{~g} / \mathrm{L} ; \mathrm{lgG} 4,0.052-1.250 \mathrm{~g} / \mathrm{L} ;$ total $\operatorname{lgG}$, 7.0-16.0 g/L. ${ }^{a}$ All participants were diagnosed with hypogammaglobulinemia (total $\operatorname{lgG}<7.0 \mathrm{~g} / \mathrm{L}$ ). 
(Table 3). Since the IgG subclass levels in our models were expressed using a negative base-2 logarithmic scale, for every one-unit increase on this $\log$ scale, the IgG subclass levels decreased by $50 \%$ (negative $\log _{2} 2=-1$ ). Thus, in MACRO, a 50\% reduction of IgG1 levels was associated with a 30\% higher adjusted HR for COPD exacerbations. A similar reduction in IgG2 levels resulted in a 19\% higher adjusted HR for this outcome. These findings were replicated in STATCOPE, as the adjusted HRs related to COPD exacerbations were 1.25 (95\% CI: $1.02-1.54, p=0.035)$ and 1.17 (95\% CI: 1.01-1.36, $p=0.046$ ) for IgG1 and IgG2, respectively. IgG1 and IgG2 levels were also associated with hospitalizations in MACRO: the adjusted HR for every 50\% reduction of IgG1 level was 1.52 (95\% CI: 1.15-2.02, $p=$ 0.004 ) and for IgG2 was 1.33 (95\% CI: $1.08-1.64, p=0.007$ ) (Table 4). In STATCOPE, only decreased IgG2 levels resulted in a higher risk of hospitalizations (adjusted HR = $1.43,95 \%$ CI: $1.12-1.83, \mathrm{p}=0.004)$. We also detected an inverse relationship between IgG levels (log-transformed) and the adjusted predicted risk of COPD exacerbations (Fig. 2) and hospitalizations (Fig. 3) in MACRO and STATCOPE cohorts ( $p<0.05$ for both outcomes).

In our multivariate Cox models, IgG3 and IgG4 levels did not result in significant associations for both outcomes in either MACRO or STATCOPE cohorts. For these two IgG subclasses, we also calculated the HRs using both cohorts combined $(n=1629)$ and obtained similar HRs compared
Table 3 Unadjusted and adjusted hazard ratios for COPD exacerbations according to lgG subclass levels in the MACRO and STATCOPE cohorts

\begin{tabular}{|c|c|c|c|c|}
\hline \multicolumn{5}{|c|}{ MACRO - Time to first Exacerbation } \\
\hline \multirow[t]{2}{*}{$\lg G^{a}$} & \multicolumn{2}{|l|}{ Univariate analysis } & \multicolumn{2}{|l|}{ Multivariate analysis ${ }^{b}$} \\
\hline & Hazard Ratio (95\% Cl) & P & Hazard Ratio $(95 \%$ Cl) & $P$ \\
\hline $\operatorname{lgG1}$ & $1.43(1.23-1.67)$ & $<0.001$ & $1.30(1.10-1.54)$ & 0.002 \\
\hline $\lg G 2$ & $1.29(1.15-1.45)$ & $<0.001$ & $1.19(1.05-1.35)$ & 0.006 \\
\hline $\lg G 3$ & $0.99(0.88-1.11)$ & 0.84 & $0.95(0.85-1.07)$ & 0.39 \\
\hline $\operatorname{lgG} 4$ & $1.09(1.02-1.17)$ & 0.009 & $1.05(0.98-1.13)$ & 0.17 \\
\hline \multicolumn{5}{|c|}{ STATCOPE - Time to first Exacerbation } \\
\hline $\lg G^{a}$ & Hazard Ratio (95\% Cl) & $P$ & Hazard Ratio $(95 \% \mathrm{Cl})$ & $P$ \\
\hline $\operatorname{lgG1}$ & $1.45(1.20-1.75)$ & $<0.001$ & $1.25(1.02-1.54)$ & 0.035 \\
\hline $\lg G 2$ & $1.32(1.15-1.52)$ & $<0.001$ & $1.17(1.01-1.36)$ & 0.046 \\
\hline $\operatorname{lgG} 3$ & $1.10(0.98-1.25)$ & 0.11 & $0.99(0.87-1.13)$ & 0.90 \\
\hline $\operatorname{lgG} 4$ & $1.01(0.94-1.08)$ & 0.76 & $0.96(0.89-1.04)$ & 0.33 \\
\hline
\end{tabular}

${ }^{\mathrm{a}} \mathrm{ggG}$ subclass levels expressed using a negative-log transformation (base 2), with one-unit increase on this log scale being equivalent to a $50 \%$ decrease of $\lg G$ subclass levels. ${ }^{b}$ Adjusted hazard ratios for IgG levels were calculated using a Cox Proportional Hazards model with adjustments for the following covariates: study drug (azithromycin vs. placebo in MACRO - First cohort; simvastatin vs. placebo in STATCOPE - Replication cohort), age, gender, ethnicity (Caucasian vs. Non-Caucasian), $\mathrm{FEV}_{1}$ (\% of predicted values), smoking status (current vs. former smokers), oxygen dependence, inhaled corticosteroid use, and treatment with systemic steroids in previous year. Legend: $95 \% \mathrm{Cl}=95 \%$ Confidence Interval
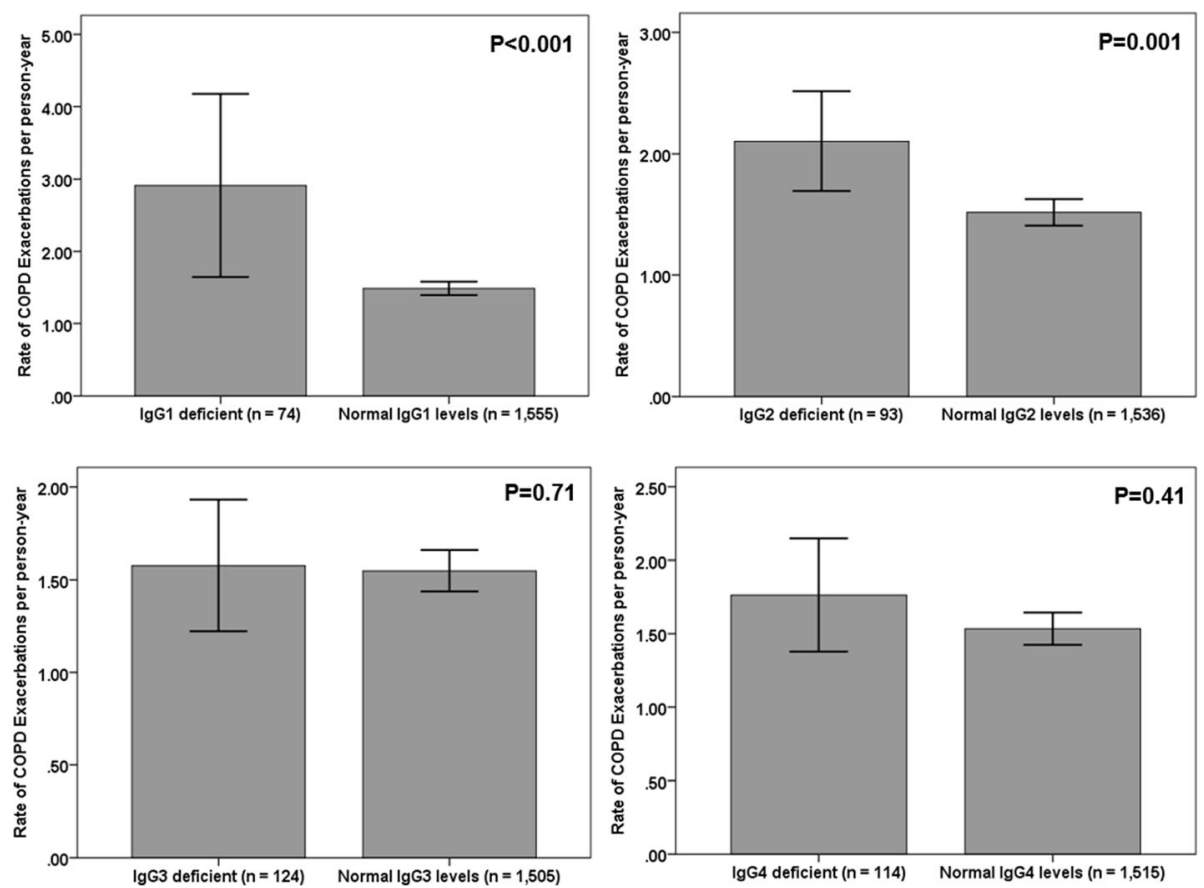

Fig. 1 Comparison of rates of COPD exacerbations in person-years between participants with lgG subclass deficiency and normal lgG subclass levels in the merged dataset (MACRO and STATCOPE cohorts combined, $n=1629$ ). Error bars represent $95 \%$ confidence interval 
Table 4 Unadjusted and adjusted hazard ratios related to hospitalizations according to lgG subclass levels in the MACRO and STATCOPE cohorts

\begin{tabular}{|c|c|c|c|c|}
\hline \multicolumn{5}{|c|}{ MACRO - Time to first Hospitalization } \\
\hline \multirow[t]{2}{*}{$\lg G^{a}$} & \multicolumn{2}{|l|}{ Univariate analysis } & \multicolumn{2}{|l|}{ Multivariate analysis ${ }^{\mathrm{b}}$} \\
\hline & Hazard Ratio (95\% Cl) & $P$ & Hazard Ratio $(95 \% \mathrm{Cl})$ & $P$ \\
\hline $\lg G 1$ & $1.51(1.16-1.99)$ & 0.003 & $1.52(1.15-2.02)$ & 0.00 \\
\hline $\lg G 2$ & $1.37(1.12-1.67)$ & 0.002 & $1.33(1.08-1.64)$ & 0.00 \\
\hline $\operatorname{lgG3}$ & $1.03(0.85-1.24)$ & 0.79 & $1.01(0.84-1.44)$ & 0.90 \\
\hline $\operatorname{lgG} 4$ & $1.01(0.89-1.12)$ & 0.99 & $1.00(0.88-1.12)$ & 0.94 \\
\hline \multicolumn{5}{|c|}{ STATCOPE - Time to first Hospitalization } \\
\hline \multirow[t]{2}{*}{$\lg G^{a}$} & \multicolumn{2}{|l|}{ Univariate analysis } & \multicolumn{2}{|l|}{ Multivariate analysis ${ }^{\mathrm{b}}$} \\
\hline & Hazard Ratio (95\% Cl) & $P$ & Hazard Ratio $(95 \% \mathrm{Cl})$ & $P$ \\
\hline $\operatorname{lgG1}$ & $1.33(0.98-1.80)$ & 0.07 & $1.27(0.91-1.76)$ & 0.16 \\
\hline $\lg G 2$ & $1.39(1.11-1.74)$ & 0.004 & $1.43(1.12-1.83)$ & 0.002 \\
\hline $\operatorname{lgG} 3$ & $0.97(0.79-1.19)$ & 0.75 & $0.90(0.73-1.12)$ & 0.36 \\
\hline $\lg G 4$ & $1.08(0.95-1.22)$ & 0.22 & $1.06(0.93-1.20)$ & 0.39 \\
\hline
\end{tabular}

${ }^{a}$ lgG subclass levels expressed using a negative-log transformation (base 2), with oneunit increase on this log scale being equivalent to a $50 \%$ decrease of lgG subclass levels. ${ }^{b}$ Adjusted hazard ratios for lgG levels were calculated using a Cox Proportional Hazards model with adjustments for the following covariates: study drug (azithromycin vs. placebo in MACRO - First cohort; simvastatin vs. placebo in STATCOPE - Replication cohort), age, gender, ethnicity (Caucasian vs. Non-Caucasian), FEV 1 (\% of predicted values), smoking status (current vs. former smokers), oxygen dependence, inhaled corticosteroid use, and treatment with systemic steroids in previous year. Legend: $95 \%$ $\mathrm{Cl}=95 \%$ Confidence Interval to analyzing the cohorts separately: IgG3 (exacerbations: $\mathrm{HR}=0.97,95 \% \mathrm{CI}: 0.89-1.05, p=0.53$; hospitalizations: $\mathrm{HR}=0.97,95 \% \mathrm{CI}: 0.84-1.12, p=0.70$ ); IgG4 (exacerbations: $\mathrm{HR}=1.01,95 \% \mathrm{CI}: 0.96-1.06, p=0.71$; hospitalizations: $1.02,95 \%$ CI: $0.94-1.12, p=0.59$ ).

We observed a trend for lower IgG1 subclass levels among participants who were treated with systemic steroids in the year prior to enrollment (MACRO - outcome: time to first exacerbation, $p=0.057$; STATCOPE outcome: time to first hospitalization, $p=0.07$ ) (Additional file 6: Table S4). No statistically significant interactions were observed between IgG subclass levels and inhaled steroid therapy in both cohorts for either exacerbations or hospitalizations.

\section{Discussion}

In this study, we found that IgG subclass deficiency was common in COPD, affecting approximately 1 in 5 patients who were at increased risk for COPD exacerbations. In addition, progressively lower IgG1 and IgG2 levels were associated with increased risk of exacerbations and hospitalizations in two different COPD cohorts. Moreover, the protective effect of IgG1 and IgG2 was even more pronounced on hospitalizations compared to exacerbations in both analyzed cohorts, emphasizing the potential role of IgG subclass levels in modulating the frequency and severity of COPD exacerbations.
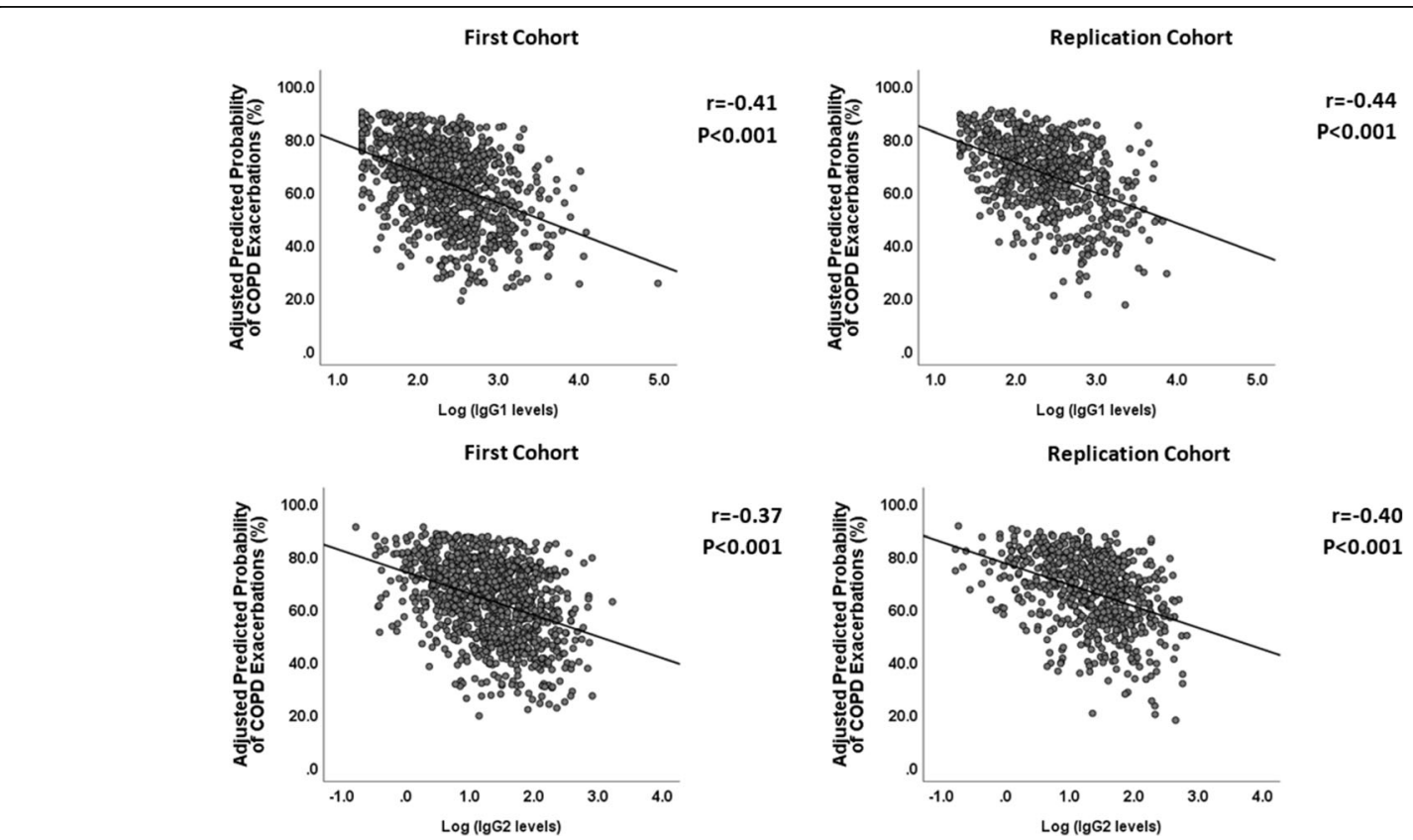

Fig. 2 Correlation of IgG1 (upper panel) and lgG2 (lower panel) levels (log-transformed, base2) and the predicted risk of COPD exacerbations. For each participant, the predicted risks were calculated using logistic regression models with adjustments for the following covariates: study drug (azithromycin vs. placebo in MACRO - First cohort; simvastatin vs. placebo in STATCOPE - Replication cohort), age, gender, ethnicity (Caucasian vs. non-Caucasian), FEV ${ }_{1}$ (\% of predicted values), smoking status (current vs. former smokers), oxygen dependence, inhaled corticosteroid use, and treatment with systemic steroids in previous year 
First Cohort

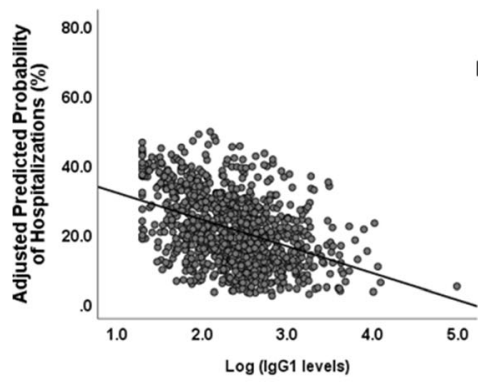

First Cohort

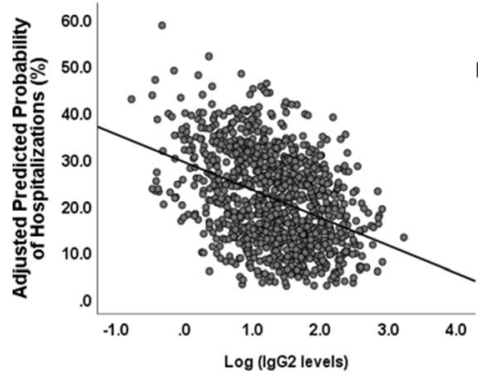

Replication Cohort

$r=-0.41$ $P<0.001$

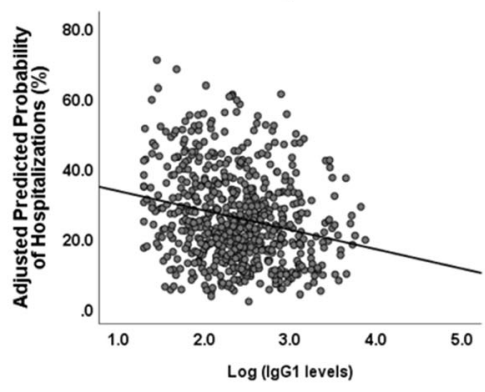

$r=-0.23$

$\mathrm{P}<0.001$

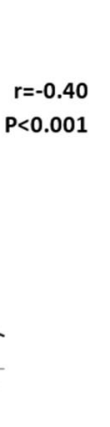

Fig. 3 Correlation of IgG1 (upper panel) and lgG2 (lower panel) levels (log-transformed, base2) and the predicted risk of hospitalizations. For each participant, the predicted risks were calculated using logistic regression models with adjustments for the following covariates: study drug (azithromycin vs. placebo in MACRO - First cohort; simvastatin vs. placebo in STATCOPE - Replication cohort), age, gender, ethnicity (Caucasian vs. non-Caucasian), FEV $1 \%$ of predicted values), smoking status (current vs. former smokers), oxygen dependence, inhaled corticosteroid use, and treatment with systemic steroids in previous year

Several prior reports have described the prevalence of IgG subclass deficiency in COPD patients [15-17]. O'Keeffe et al. observed an IgG subclass deficiency in 15 of 58 COPD patients (25.9\%) [15]. Qvarfordt et al. measured IgG subclass levels in 33 smokers with chronic bronchitis and recurrent exacerbations, identifying an IgG subclass deficiency in 6 patients (18\%) [16]. We obtained similar results, as at least one IgG subclass deficiency was detected in $17.7 \%$ and $20.4 \%$ of participants with COPD in MACRO and STATCOPE cohorts, respectively. Concerning the distribution of IgG subclass deficiency, we identified $\operatorname{IgG3}$ as the most frequent (6.7\% in MACRO and $9.0 \%$ of cases in STATCOPE), which has also been reported by other studies $[16,23]$. Our findings suggest that the frequency of IgG abnormalities may be even higher among COPD patients who present with multiple exacerbations, as we detected a higher frequency of IgG1 and IgG2 deficiency among recurrent exacerbators. This is supported by a previous article, in which IgA, IgG, and IgM were measured in 42 COPD patients who had 2 or more moderate to severe exacerbations per year [24]. The authors detected in 29 patients (69\%) evidence of an antibody deficiency syndrome, with 8 patients receiving a diagnosis of common variable immunodeficiency syndrome, whose diagnosis requires reduced total IgG levels [25]. Similarly, we observed significantly lower IgG1 and IgG2 levels among COPD participants who developed exacerbations and required hospitalizations in both cohorts.

In our Cox proportional hazards regression models, reductions in IgG1 and IgG2 levels in the First cohort were associated with an increased risk of COPD exacerbations and hospitalizations. These results remained statistically significant after adjustments for differences in age, gender, race, study drug, smoking status, oxygen dependence, $\mathrm{FEV}_{1}$ (\% of predicted values), use of inhaled steroids and treatment with systemic steroids in the year prior to enrollment. Similar results were detected using a second independent cohort (thus increasing the validity of our findings) [26], with the exception that only IgG2 remained as an independent predictor of hospitalizations. Additionally, we also showed that participants with IgG1 and/or IgG2 deficiency presented with higher rates of COPD exacerbations per person-year during follow-up. To the best of our knowledge, this is the first report to demonstrate that lower IgG subclass levels are independent risk factors for both COPD exacerbations (IgG1 and IgG2) and hospitalizations (IgG2).

Our results are in line with some important concepts related to the biology of IgG subclasses. IgG1 and IgG2 are the major components of serum total IgG levels, explaining why patients with deficiencies in either one of these subclasses (or both) are more likely to present with reduced total IgG 
levels (i.e., hypogammaglobulinemia) [10, 11, 25]. We observed that $97.3 \%$ and $74.2 \%$ of patients diagnosed with IgG1 and IgG2 deficiencies (considering the merged cohort) also met criteria for hypogammaglobulinemia, respectively. Thus, patients with lower IgG1 and IgG2 levels, through a reduction in total IgG levels, may present with an increased risk of COPD exacerbations and hospitalizations, as these outcomes are mostly triggered by respiratory infections [4]. This is consistent with a previous work of our group, in which COPD patients with hypogammaglobulinemia showed a higher frequency of COPD exacerbations and hospitalizations compared to patients with normal total IgG levels [14]. Additionally, patients with IgG2 deficiency usually present with impaired polysaccharide responses, leading to a higher susceptibility to infections caused by encapsulated pathogens (e.g., Streptococcus pneumoniae and Haemophilus influenzae type B) $[10,12,13]$, which are frequently implicated in bacterial COPD exacerbations [4].

It has been shown that not all patients with lower IgG subclasses present with recurrent infections, with some healthy individuals also being diagnosed with one or more IgG subclasses deficiencies [11]. Conversely, there is a rationale for evaluating patients with recurrent infections for possible IgG subclass deficiency. Kim JH et al. observed that one-third of patients with chronic airway diseases (asthma, COPD, or asthma COPD overlap syndrome) who had received a previous diagnosis of IgG subclass deficiency $(n=59)$ presented with a history of recurrent respiratory infections [23]. Additionally, these patients showed a higher frequency of hospitalizations and a faster $\mathrm{FEV}_{1}$ decline during follow-up. Our results indicate that IgG1 or IgG2 subclass deficiency may contribute to a higher exacerbation risk, and thus we advocate that measurement of IgG subclass levels be considered in any COPD patient with a history of several exacerbations or previous hospitalizations. Moreover, in this scenario, even if a COPD patient shows normal total IgG levels, it is still possible that an IgG1 or IgG2 subclass deficiency may be present, potentially contributing to an increased propensity to COPD exacerbations. In a previous article, Vendrell et al. found that $11 \%$ of patients with bronchiectasis of unknown etiology with normal total IgG levels $(n=107)$ still had an antibody production deficiency, with $50 \%$ of those presenting with IgG2 deficiency [27].

The management of IgG subclass deficiency includes vaccinations, prophylactic antibiotics, treatment of allergy and sinopulmonary disease (if present) and cautious use of immunoglobulin G replacement therapy - IVIG (for patients who persist with recurrent infections despite these interventions) [25]. In one previous study, administration of IVIG reduced the risk of respiratory infections by over $50 \%$ in 92/132 adults with IgG subclass deficiency presenting with recurrent infections [28]. In another study, IVIG significantly decreased the frequency of moderate to severe exacerbations by nearly $90 \%$ in 14 COPD patients (from an annual COPD exacerbation rate of $4.7 \pm 3.1$ to $0.6 \pm 1.0$ per patient)[29]. Interestingly, $64.3 \%$ of the participants (9/14) had evidence of hypogammaglobulinemia.

Our study had several limitations. Firstly, we were only able to measure IgG subclass levels at baseline, and thus we cannot determine how IgG subclass levels fluctuate over the course of COPD or with the implementation of various COPD treatments. Secondly, we only measured IgG subclass levels once, despite standard laboratory recommendations that two measurements be performed at least 1 month apart to confirm deficiency $[11,25]$. Thirdly, we did not investigate whether patients with IgG subclass deficiency had impaired humoral immunity, usually evaluated by measuring antibody levels after a pneumococcal vaccination $[11,13,25]$. Finally, since this is a retrospective, observational study, we can only describe an association between low serum IgG1 or IgG2 levels and COPD exacerbations. Although IgG subclass deficiency may serve as a biomarker to identify subjects at increased risk of exacerbation, our study was not designed to determine if low serum IgG subclass levels are linked on a causal pathway to COPD exacerbations. It is worth mentioning we analyzed samples from moderate-to-severe COPD patients who were at increased risk of COPD exacerbations; thus the effect of lower IgG levels on the outcomes of COPD patients with mild disease or without a history of prior exacerbations was not evaluated by our study.

\section{Conclusions}

Our data support that IgG subclass deficiency is relatively common among COPD patients and that decreased IgG1 and IgG2 levels result in an increased risk of adverse outcomes in COPD. Future prospective studies are needed to better elucidate the impact of these IgG subclass deficiencies on the management of high-risk COPD patients and whether they represent a modifiable risk factor for COPD exacerbations and hospitalizations through IVIG.

\section{Additional files}

Additional file 1: Table S1. Prevalence of IgG subclass deficiencies in MACRO and STATCOPE cohorts. (DOCX $15 \mathrm{~kb}$ )

Additional file 2: Table S2. Median and interquartile range related to each IgG subclass according to the presence or absence of corresponding IgG subclass deficiency in MACRO and STATCOPE cohorts. (DOCX 15 kb)

Additional file 3: Table S3. Median and interquartile range related to each IgG subclass according to the presence or absence of corresponding lgG subclass deficiency in the merged dataset (MACRO and STATCOPE cohorts combined). (DOCX $15 \mathrm{~kb}$ )

Additional file 4: Figure S1. Comparison of lgG subclass levels according to exacerbation status in MACRO - First cohort (left panel) and STATCOPE - Replication cohort (right panel). Error bars represent 95\% confidence interval. (DOCX 205 kb)

Additional file 5: Figure S2. Comparison of lgG subclass levels according to hospitalization status in MACRO - First cohort (left panel) and STATCOPE - Replication cohort (right panel) cohorts. Error bars represent 95\% confidence interval. (DOCX 243 kb) 
Additional file 6: Table S4. Interactions between lgG subclass levels and inhaled steroid use at enrollment and use of systemic steroids in previous 12 months for both outcomes of interest (time to first exacerbation and time to first hospitalization). (DOCX 15 kb)

\section{Abbreviations}

$\mathrm{Cl}$ : Confidence intervals; COPD: Chronic obstructive pulmonary disease; $\mathrm{FEV}_{1}$ : Forced expiratory volume in $1 \mathrm{~s}$; HR: Hazard ratio; Ig: Immunoglobulins; IgG: Immunoglobulin G; IVIG: Immunoglobulin G replacement therapy; LLN: Lower limit of normal; MACRO: Macrolide Azithromycin for Prevention of Exacerbations of COPD trial; STATCOPE: Simvastatin for the Prevention of Exacerbations in Moderate-to-Severe COPD trial

\section{Acknowledgments}

We thank the COPD Clinical Research Network for conducting both the MACRO and STATCOPE trials and for providing us access to all available samples in both cohorts. Dr. Leitao Filho FS gratefully acknowledges postdoctoral support from CNPq-Brazil and the Canadian Institutes of Health Research (CIHR) Integrated and Mentored Pulmonary and Cardiovascular Training program (IMPACT).

\section{Funding}

This work was supported by the BC Lung Association and the Canadian Respiratory Research Network (CRRN). The MACRO and STATCOPE trials were funded by the US National Heart Blood and Lung Institute (NHLBI).

\section{Availability of data and materials}

The datasets used and/or analyzed during the current study are available from the corresponding author on reasonable request.

\section{Authors' contributions}

FSLF, SWR, RMR, SDA, RSS and DDS: conception and design of the work; RMR, SDA, AM, RA, GJC, PGW, JEC, SCL, MKH, FJM, and DDS: Acquisition of data; All authors: analysis and interpretation of data; FSLF, SWR, SDA, RMR, RSS and DDS: Drafting the manuscript; All authors: Review and approval of the final version of the manuscript for important intellectual content.

\section{Ethics approval and consent to participate}

Both trials received approval from each participating institutions' local research ethics board. This analysis received approval from the University of British Columbia/ Providence Health Care Research Ethics Committee (Approval No. H16-03232).

\section{Consent for publication}

Not applicable.

\section{Competing interests}

The authors declare that they have no competing interests.

\section{Publisher's Note}

Springer Nature remains neutral with regard to jurisdictional claims in published maps and institutional affiliations.

\section{Author details}

${ }^{1}$ Centre for Heart Lung Innovation, St. Paul's Hospital, Vancouver, BC V6Z 1Y6, Canada. ${ }^{2}$ Department of Medicine (Division of Respiratory Medicine), University of British Columbia, Vancouver, BC, Canada. ${ }^{3}$ Federal University of Ceará, Fortaleza, Ceará, Brazil. ${ }^{4}$ University of Ulsan College of Medicine, Ulsan University Hospital, Ulsan, South Korea. ${ }^{5}$ Department of Pathology and Laboratory Medicine, University of British Columbia, Vancouver, BC, Canada. ${ }^{6}$ Department of Thoracic Medicine and Surgery, Lewis Katz school of Medicine at Temple University, Philadelphia, PA, USA. 'Department of Medicine, University of California San Francisco, San Francisco, CA, USA. ${ }^{8}$ Pulmonary Sciences and Critical Care Medicine, University of Colorado, Denver, CO, USA. ${ }^{9}$ School of Public Health, University of Minnesota, Minneapolis, MN, USA. ${ }^{10}$ Department of Internal Medicine, University of Michigan, Ann Arbor, MI, USA. ${ }^{11}$ Joan and Sanford I. Weill Department of Medicine, Weill Cornell Medical College, Cornell University, New York, NY, USA. ${ }^{12}$ Department of Medicine, University of Ottawa, Ottawa, ON, Canada. ${ }^{13}$ Division of Pulmonary and Critical Care Medicine, University of Maryland School of Medicine, Baltimore, MD, USA.
Received: 15 January 2018 Accepted: 31 January 2018

Published online: 14 February 2018

\section{References}

1. Vogelmeier CF, Criner GJ, Martinez FJ, Anzueto A, Barnes PJ, Bourbeau J, et al. Global Strategy for the Diagnosis, Management, and Prevention of Chronic Obstructive Lung Disease 2017 Report. GOLD Executive Summary. Am. J. Respir. Crit. Care Med. 2017;195(5):557-82.

2. Anzueto A. Impact of exacerbations on COPD. Eur. Respir. Rev. 2010;19(116):113-8.

3. Soler-Cataluna JJ, Martinez-Garcia MA, Roman Sanchez P, Salcedo E, Navarro M, Ochando R. Severe acute exacerbations and mortality in patients with chronic obstructive pulmonary disease. Thorax. 2005;60(11):925-31.

4. Miravitlles $M$, Anzueto A. Role of infection in exacerbations of chronic obstructive pulmonary disease. Curr. Opin. Pulm. Med. 2015;21(3):278-83.

5. Umetsu DT, Ambrosino DM, Quinti I, Siber GR, Geha RS. Recurrent sinopulmonary infection and impaired antibody response to bacterial capsular polysaccharide antigen in children with selective lgG-subclass deficiency. N. Engl. J. Med. 1985;313(20):1247-51.

6. Aucouturier P, Lacombe C, Bremard C, Lebranchu Y, Seligmann M, Griscelli $C$, et al. Serum IgG subclass levels in patients with primary immunodeficiency syndromes or abnormal susceptibility to infections. Clin. Immunol. Immunopathol. 1989;51(1):22-37.

7. Popa V, Kim K, Heiner DC. IgG deficiency in adults with recurrent respiratory infections. Ann. Allergy. 1993;70(5):418-24.

8. Popa V. Airway obstruction in adults with recurrent respiratory infections and lgG deficiency. Chest. 1994;105(4):1066-72.

9. Sethi S, Murphy TF. Infection in the pathogenesis and course of chronic obstructive pulmonary disease. N. Engl. J. Med. 2008;359(22):2355-65.

10. Schroeder HW Jr, Cavacini L. Structure and function of immunoglobulins. J. Allergy Clin. Immunol. 2010;125(2 Suppl 2):S41-52.

11. Agarwal S, Cunningham-Rundles C. Assessment and clinical interpretation of reduced IgG values. Ann. Allergy Asthma Immunol. 2007;99(3):281-3.

12. Vidarsson $G$, Dekkers $G$, Rispens T. IgG subclasses and allotypes: from structure to effector functions. Front Immunol. 2014;5:520.

13. Maguire GA, Kumararatne DS, Joyce HJ. Are there any clinical indications for measuring lgG subclasses? Ann. Clin Biochem. 2002;39(Pt 4):374-7.

14. Leitao Filho FS, Won Ra S, Mattman A, Schellenberg RS, Fishbane N, Criner GJ, et al. Serum IgG and risk of exacerbations and hospitalizations in chronic obstructive pulmonary disease. J. Allergy Clin. Immunol. 2017;140(4):1164-7. e6

15. O'Keeffe S, Gzel A, Drury R, Cullina M, Greally J, Finnegan P. Immunoglobulin $\mathrm{G}$ subclasses and spirometry in patients with chronic obstructive pulmonary disease. Eur. Respir. J. 1991;4(8):932-6.

16. Qvarfordt I, Riise GC, Andersson BA, Larsson S. IgG subclasses in smokers with chronic bronchitis and recurrent exacerbations. Thorax. 2001;56(6):445-9.

17. Karnak D, Beder S, Kayacan O, Karaca L. IgG Subclass Levels in ELF in COPD. Turk J Med Sci. 2001;31:235-41.

18. Zhou A, Zhou Z, Zhao Y, Chen $P$. The recent advances of phenotypes in acute exacerbations of COPD. Int J Chron Obstruct Pulmon Dis. 2017;12: 1009-18.

19. Albert RK, Connett J, Bailey WC, Casaburi R, Cooper JA Jr, Criner GJ, et al. Azithromycin for prevention of exacerbations of COPD. N. Engl. J. Med. 2011;365(8):689-98.

20. Criner GJ, Connett JE, Aaron SD, Albert RK, Bailey WC, Casaburi R, et al. Simvastatin for the prevention of exacerbations in moderate-to-severe COPD. N. Engl. J. Med. 2014;370(23):2201-10.

21. Schauer U, Stemberg F, Rieger $\mathrm{CH}$, Borte M, Schubert S, Riedel F, et al. IgG subclass concentrations in certified reference material 470 and reference values for children and adults determined with the binding site reagents. Clin. Chem. 2003:49(11):1924-9.

22. Stiehm ER, Ochs HD, Winkelstein JA. Immunologic Disorders in Infants and Children. 5th ed. Philadelphia: Saunders; 2004. p. 1512.

23. Kim JH, Park S, Hwang YI, Jang SH, Jung KS, Sim YS, et al. Immunoglobulin G Subclass Deficiencies in Adult Patients with Chronic Airway Diseases. J. Korean Med. Sci. 2016;31(10):1560-5.

24. McCullagh BN, Comellas AP, Ballas ZK, Newell JD Jr, Zimmerman MB, Azar $A E$. Antibody deficiency in patients with frequent exacerbations of Chronic Obstructive Pulmonary Disease (COPD). PloS One. 2017;12(2):e0172437.

25. Bonilla FA, Khan DA, Ballas ZK, Chinen J, Frank MM, Hsu JT, et al. Practice parameter for the diagnosis and management of primary immunodeficiency. J. Allergy Clin. Immunol. 2015;136(5):1186-205 e1-78. 
26. Carlson MD, Morrison RS. Study design, precision, and validity in observational studies. J. Palliat. Med. 2009;12(1):77-82.

27. Vendrell M, de Gracia J, Rodrigo MJ, Cruz MJ, Alvarez A, Garcia M, et al. Antibody production deficiency with normal lgG levels in bronchiectasis of unknown etiology. Chest. 2005;127(1):197-204.

28. Olinder-Nielsen AM, Granert C, Forsberg P, Friman V, Vietorisz A, Bjorkander J. Immunoglobulin prophylaxis in 350 adults with IgG subclass deficiency and recurrent respiratory tract infections: a long-term follow-up. Scand. J. Infect. Dis. 2007;39(1):44-50.

29. Cowan J, Gaudet L, Mulpuru S, Corrales-Medina V, Hawken S, Cameron C, et al. A Retrospective Longitudinal Within-Subject Risk Interval Analysis of Immunoglobulin Treatment for Recurrent Acute Exacerbation of Chronic Obstructive Pulmonary Disease. PloS One. 2015;10(11):e0142205.

Submit your next manuscript to BioMed Central and we will help you at every step:

- We accept pre-submission inquiries

- Our selector tool helps you to find the most relevant journal

- We provide round the clock customer support

- Convenient online submission

- Thorough peer review

- Inclusion in PubMed and all major indexing services

- Maximum visibility for your research

Submit your manuscript at www.biomedcentral.com/submit
Biomed Central 